Proyecciones

Vol. 19, No 3, pp. 271-289, December 2000.

Universidad Católica del Norte

Antofagasta - Chile

\title{
DISEÑO ÓPTIMO Y HOMOGENIZACIÓN
}

\author{
SERGIO GUTIÉRREZ \\ Pontificia Universidad Católica de Chile, Chile
}

\begin{abstract}
Este artículo pretende dar una mayor difusión al uso de la teoría general de homogenización, desarrollada por F. Murat, L. Tartar y otros autores, en conexión con el diseño óptimo de materiales compuestos. El poder y la relativa simplicidad de la teoría son enfatizados mostrando varios resultados conocidos para los problemas de difusión y de elasticidad lineal.
\end{abstract}




\section{Introducción}

El término "diseño óptimo" es utilizado para agrupar distintas herramientas matemáticas que ayudan a estudiar el problema de optimizar el diseño de una componente de una estructura, por medio de variar, por ejemplo, su geometría como en el diseño de perfiles de alas de aviones, o la manera de mezclar a escala muy pequeña distintos materiales básicos, como es en el caso de los materiales compuestos. El estudio de los materiales compuestos ha suscitado el interés de científicos e ingenieros de varias especialidades desde hace mucho tiempo, pero desde mediados de este siglo ha ido concitando cada vez más interés, debido, por un lado a las crecientes exigencias en cuanto al comportamiento de los materiales utilizados en las aplicaciones convencionales, pero también a la necesidad de diseñar materiales ad-hoc para las nuevas aplicaciones hechas posibles con el avance de la tecnología, todo lo cual ha contribuído a la consolidación de la ciencia de los materiales como una especialidad por sí sola. Sin embargo la complejidad matemática involucrada en el problema de predecir el comportamiento de los materiales compuestos, debida en parte al hecho de tener que combinar mediciones de órdenes de magnitud muy distintos, llevó a que muchas veces las fórmulas utilizadas fueran simplemente empíricas o bien hayan sido derivadas sin mayor rigurosidad matemática. En las últimas dos o tres décadas, sin embargo, la teoría matemática que se preocupa de estas preguntas, la teoría de homogenización, ha tenido un gran desarrollo, lo que ha llevado a la aparición de varias ideas en cuanto a la utilización de convergencias débiles adaptadas al estudio de estos problemas. Entre ellas podemos mencionar la idea de la G-convergencia (Spagnolo [14]), la H-convergencia (Murat [11] y Tartar [15]), la $\Gamma$-convergencia (Dal Maso [3]) y el uso de las medidas de Young (Ball [2] y Pedregal [13]), por citar tan solo las referencias más usuales.

La teoría de homogenización proporciona la noción de convergencia H, introducida por François Murat y Luc Tartar, que en un contexto bastante general nos permite tomar el límite de una sucesión de ecuaciones válidas a una escala muy pequeña, para obtener información a escala macroscópica y así poder predecir cómo se comportará el material compuesto a la escala de los fenómenos físicos más 
usuales. Un material laminado es un compuesto tal que a escala muy pequeña está constituído por láminas alternadas de dos materiales homogéneos, pero que a escala macroscópica se comporta como si fuera homogéneo. Para materiales laminados, la convergencia H nos permite obtener fórmulas explícitas para el tensor de difusión de calor efectivo u homogenizado y, bajo ciertos supuestos matemáticos, también para el tensor de elasticidad. En la referencia [15] Luc Tartar presentó por primera vez los resultados fundamentales de homogenización aquí utilizados, sin embargo es la referencia [17] la que contiene todos los detalles de los resultados aquí utilizados.

En el caso del cálculo del tensor efectivo de elasticidad, los supuestos matemáticos arriba señalados son, en principio, demasiado restrictivos, dado que no permiten trabajar con la clase de materiales elásticos más general posible. Sin embargo, se pueden construír ejemplos que muestran que para el sistema de elasticidad lineal, la clase más general es demasiado grande y, que en el mejor de los casos, uno sólo podría relajar "levemente" los supuestos matemáticos antes mencionados, para mantener consistencia con lo esperado físicamente.

Con respecto a la notación, denotaremos por $N$ a la dimensión del espacio físico donde trabajamos, por tanto $N$ tomará los valores 1, 2 ó 3. Los exponentes $n$ deben siempre entenderse como índices de una sucesión y cuando sea necesario extraer una subsucesión, ésta será reindexada de manera de denotarla como la sucesión original, pues de ahí en adelante nuestra atención se ocupará sólo de la subsucesión extraída. $\mathcal{M}_{N}$ será el espacio de las matrices cuadradas de orden $N$ y $S y m_{N}$ será el subespacio de las matrices simétricas de orden $N$. $\Omega$ será un abierto acotado de frontera regular en $\mathbb{R}^{N}$. $L^{\infty}(\Omega)$ es el espacio de las funciones (esencialmente) acotadas en $\Omega, L^{2}(\Omega)$ es el espacio de las funciones definidas en $\Omega$ tales que su cuadrado es una función integrable en $\Omega$, el cual es un espacio de Hilbert con producto interno

$$
(f, g)=\int_{\Omega} f(x) g(x) d x
$$

$H^{1}(\Omega)$ es el espacio de las funciones definidas en $\Omega$ tales que su cuadrado es una función integrable en $\Omega$ y también todas sus primeras derivadas parciales son de cuadrado integrable en $\Omega$. $H^{1}(\Omega)$ es también un es- 
pacio de Hilbert con producto interno

$$
(f, g)=\int_{\Omega}\left(f(x) g(x)+\sum_{i=1}^{N} \frac{\partial f}{\partial x_{i}} \frac{\partial g}{\partial x_{i}}\right) d x .
$$

$H_{0}^{1}(\Omega)$ es el subespacio de $H^{1}(\Omega)$ que contiene solamente a las funciones que toman el valor cero en el borde de $\Omega$, denotado $\partial \Omega$. La notación $L^{2}\left(\Omega, \mathbf{R}^{N}\right)$ se usará para representar a las funciones vectoriales definidas en $\Omega$ y tales que todas sus componentes están en $L^{2}(\Omega)$, análogamente $L^{2}\left(\Omega, \mathcal{M}_{N}\right), H^{1}\left(\Omega, \mathbf{R}^{N}\right)$, etc. $H^{-1}(\Omega)$ es el espacio dual topológico de $H_{0}^{1}(\Omega)$, es decir contiene a todas las funciones lineales continuas de $H_{0}^{1}(\Omega)$ en $\mathbb{R}$.

Si $H$ es un espacio de Banach cualquiera, se denotará a su dual topológico como $H^{\prime}$ y se dice que $u_{n}$ converge débilmente a $u_{\infty}$ en $H$, denotado $u_{n} \rightarrow u_{\infty}$ si se tiene que

$$
L\left(u_{n}\right) \rightarrow L\left(u_{\infty}\right) \quad \text { para todo } \quad L \in H^{\prime}
$$

Se dice que $L_{n}$ converge débil $\star$ a $L_{\infty}$ en $H^{\prime}$, si se tiene que

$$
L_{n}(u) \rightarrow L_{\infty}(u) \quad \text { para todo } \quad u \in H
$$

Si en un espacio de Hilbert $H$ se tiene que $u_{n} \rightarrow u_{\infty}$ débil en $H$ y $L_{n} \rightarrow L_{\infty}$ en $H^{\prime}$, se tendrá que $L_{n}\left(u_{n}\right) \rightarrow L_{\infty}\left(u_{\infty}\right)$. Si se tiene que $\left\{u_{n}\right\}$ es una sucesión acotada en $H$, ésta necesariamente tendrá una subsucesión débilmente convergente en $H$.

A menos que se diga lo contrario las afirmaciones que se hacen son válidas para cualquier dimensión espacial $N=1,2$ ó 3, con las adaptaciones correspondientes.

\section{La Teoría General de Homogenización}

La idea de la teoría de homogenización es poder determinar las ecuaciones dife-renciales que describen un cierto fenómeno a escala macroscópica, partiendo de las ecuaciones diferenciales válidas a nivel microscópico, pero esto significa una escala mucho mayor que la escala atómica, pues se requiere que sean válidas las ecuaciones diferenciales obtenidas usando la teoría de medios continuos. 
- Definición de convergencia H :

Sea $\left\{A^{n}\right\}$ una sucesión en sediráqueA ${ }^{n}$ converge en sentido $\mathrm{H}$ a $A^{e f}$ si para todo $f \in H^{-1}(\Omega)$, la sucesión de soluciones $\left\{u_{n}\right\} \subset H_{0}^{1}(\Omega)$, de los problemas

$$
\left.\begin{array}{rc}
-\operatorname{div}:\left(A^{n}(x): \operatorname{grad} u_{n}(x):\right)=f(x) & x \in \Omega \\
u_{n}(x)=0 & x \in \partial \Omega,
\end{array}\right\}
$$

satisface que $u_{n} \rightarrow u_{\infty}$ débilmente en $H_{0}^{1}(\Omega)$ y también $A^{n}(x)$ : $\operatorname{grad} u_{n} \rightarrow A^{\text {ef }}(x): \operatorname{grad} u_{\infty}$ débilmente en $L^{2}\left(\Omega, \mathbf{R}^{N}\right)$, donde $u_{\infty}$ es solución del problema siguiente

$$
\left.\begin{array}{rc}
-\operatorname{div}:\left(A^{e f}(x): \operatorname{grad} u_{\infty}(x):\right)=f(x) & x \in \Omega \\
u_{\infty}(x)=0 & x \in \partial \Omega .
\end{array}\right\}
$$

Si existen $\beta>\alpha>0$ tales que para casi todo $x \in \Omega$

$$
(A(x) \xi, \xi) \geq \alpha\|\xi\|^{2} \quad \text { y } \quad(A(x) \xi, \xi) \geq \frac{1}{\beta}\|A \xi\|^{2} \quad \text { para todo } \quad \xi \in \mathbf{R}^{N}
$$

se dirá que $A \in \mathcal{M}(\alpha, \beta, \Omega)$.

\section{- Teorema 1:}

$\mathrm{M}(\alpha, \beta, \Omega)$ es secuencialmente compacto para la convergencia $\mathrm{H}$.

Esquema de la demostración:

Sea $\left\{A^{n}\right\}$ una sucesión en $\mathcal{M}(\alpha, \beta, \Omega), F$ un conjunto numerable denso en $H^{-1}(\Omega)$ y $f \in F \cap L^{2}(\Omega)$. Sea también la sucesión de los $u_{n}$ tales que

$$
\left.\begin{array}{rc}
-\operatorname{div}:\left(A^{e f}(x): \operatorname{grad} u_{n}(x):\right)=f(x) & x \in \Omega \\
u_{n}(x)=0 & x \in \partial \Omega .
\end{array}\right\}
$$

entonces

$$
-\int_{\Omega} \operatorname{div}:\left(A^{n}(x): \operatorname{grad} u_{n}(x):\right) u_{n}(x) d x=\int_{\Omega} f(x) u_{n}(x) d x
$$

y luego

$$
\int_{\Omega}\left(A^{n}(x): \operatorname{grad} u_{n}(x),: \operatorname{grad} u_{n}(x)\right) d x=\int_{\Omega} f(x) u_{n}(x) d x,
$$


pero entonces

$$
\alpha \int_{\Omega}\left(\operatorname{grad} u_{n}(x),: \operatorname{grad} u_{n}(x)\right) d x \leq \int_{\Omega} f(x) u_{n}(x) d x \leq\|f\|_{L^{2}}\left\|u_{n}\right\|_{L^{2}} .
$$

Por tanto, usando la desigualdad de Poincaré, se tendrá que

$$
\left\|u_{n}\right\|_{H^{1}} \leq M
$$

y luego existe una subsucesión de $\left\{u_{n}\right\}$ que converge débilmente en $H^{1}$ a $u_{n}(f)$.

De la definición de $\mathcal{M}(\alpha, \beta, \Omega)$ se tiene que

$$
\left\|A^{n}: \operatorname{grad} u_{n}\right\|_{L^{2}} \leq \beta\left\|\operatorname{grad} u_{n}\right\|_{L^{2}} \leq M_{1}
$$

y por tanto también existirá una subsucesión de $\left\{A^{n}: \operatorname{grad} u_{n}\right\}$ que converge débilmente en $L^{2}$.

Dado que $F$ es numerable uno puede seguir extrayendo subsucesiones de la sucesión $\left\{A^{n}\right\}$, de forma tal que $u_{n} \rightarrow u_{\infty}(f)$ en $H^{1}$ y $A^{n}: \operatorname{grad} u_{n} \rightarrow R\left(u_{\infty}(f)\right)$ en $L^{2}\left(\Omega, \mathbb{R}^{N}\right)$, para todo $f \in F \cap L^{2}(\Omega)$.

Finalmente puede demostrarse que $R: H^{1}(\Omega) \rightarrow L^{2}\left(\Omega, \mathbb{R}^{N}\right)$ es lineal continua y tal que $R\left(u_{\infty}\right)=A_{\infty} \operatorname{grad} u_{\infty}$ con $A_{\infty} \in \mathcal{M}(\alpha, \beta, \Omega)$ y por tanto la subsucesión converge en sentido $\mathrm{H}$ a $A_{\infty}$.

Para un campo vectorial regular llamaremos curl a su rotacional.

\section{- Div-Curl lema (F. Murat - L. Tartar):}

Sean $\left\{E^{n}\right\}$ y $\left\{D^{n}\right\}$ sucesiones que convergen débilmente en $L^{2}\left(\Omega, \mathbb{R}^{N}\right)$ con $E^{n} \rightarrow E^{\infty}$ y $D^{n} \rightarrow D^{\infty}$. Si además existen conjuntos $K_{1} \subset H^{-1}(\Omega)$ y $K_{2} \subset H^{-1}\left(\Omega, \mathbb{R}^{N}\right)$ compactos para las respectivas topologías fuertes, tales que para todo $n$

$$
\operatorname{div} D^{n} \in K_{1} \quad y \quad \operatorname{curl} E^{n} \in K_{2},
$$

se tendrá que existen subsucesiones tales que

$$
\lim _{n \rightarrow \infty}\left(D^{n}, E^{n}\right)=\left(D^{\infty}, E^{\infty}\right)
$$


en el sentido de las medidas, es decir en el espacio dual de $C_{c}(\Omega)$, el espacio de las funciones continuas con soporte compacto en $\Omega$.

Esquema de la demostración:

Sea $\varphi \in C_{c}^{1}(\Omega)$ y supongamos primero que $E^{n}=\operatorname{grad} u_{n}$ con $u_{n} \rightarrow u_{\infty}$ en $H^{1}$. Entonces

$$
\int_{\Omega}\left(E^{n}, D^{n}\right) \varphi d x=\int_{\Omega} \sum_{i=1}^{N} \frac{\partial u_{n}}{\partial x_{i}} D_{i}^{n} \varphi d x
$$

$=-\int_{\Omega} u_{n} \varphi \operatorname{div} D^{n} d x-\int_{\Omega}\left(D^{n}, u_{n} \operatorname{grad} \varphi\right) d x$ pero se puede extraer una subsucesión tal que $\operatorname{div} D^{n} \rightarrow \operatorname{div} D^{\infty}$ fuertemente en $H^{-1}$ y como $u_{n} \varphi \rightarrow u_{\infty} \varphi$ en $H_{0}^{1}$, se tiene que

$$
\int_{\Omega} u_{n} \varphi \operatorname{div} D^{n} d x \rightarrow \int_{\Omega} u_{\infty} \varphi \operatorname{div} D^{\infty} d x
$$

$=-\int_{\Omega}\left(E^{\infty}, D^{\infty}\right) \varphi d x-\int_{\Omega}\left(D^{\infty}, u_{\infty} \operatorname{grad} \varphi\right) d x . \quad$ Dadoque $\mathrm{H}^{1}$ se inyecta compactamente en $L^{2}$ se tiene para una subsucesión que $u_{n} \rightarrow$ $u_{\infty}$ fuertemente en $L^{2}$ y como $D^{n} \rightarrow D^{\infty}$ en $L^{2}$, se tiene entonces que

$$
\int_{\Omega}\left(D^{n}, u_{n} \operatorname{grad} \varphi\right) d x \rightarrow \int_{\Omega}\left(D^{\infty}, u_{\infty} \operatorname{grad} \varphi\right) d x
$$

Por tanto

$$
\int_{\Omega}\left(E^{n}, D^{n}\right) \varphi d x \longrightarrow \int_{\Omega}\left(E^{\infty}, D^{\infty}\right) \varphi d x
$$
$C_{c}(\Omega)$.

Luego por densidad se puede extender este argumento a $\varphi \in$

Finalmente, el caso en que $E^{n}$ no es necesariamente una sucesión de gradientes puede hacerse discretizando $\Omega$ en pequeños pedazos, en cada uno de los cuales $E^{n}$ puede escribirse como un gradiente más un pequeño error.

El Div-Curl lema es un caso particular de la técnica de Compacidad Compensada desarrollada por Tartar, siendo [16] la referencia clásica al respecto. 


\section{- Sucesión no oscilante:}

Para una sucesión $\left\{\varphi_{n}\right\}$ que converge débilmente en $L^{2}(\Omega)$ a $\varphi_{\infty}$, diremos que es no oscilante con respecto a $x_{1}$ si para toda sucesión convergente en $L^{\infty}$ débil $\star, g_{n}\left(x_{1}\right) \rightarrow g_{\infty}\left(x_{1}\right)$, se tiene que $\varphi_{n} g_{n} \rightarrow \varphi_{\infty} g_{\infty}$ débilmente en $L^{2}(\Omega)$.

Si se tiene que $A^{n}$ converge a $A^{e f}$ en el sentido $\mathrm{H}$, entonces para la sucesión de soluciones $\left\{u_{n}\right\}$ de los problemas asociados, existirá una subsucesión débilmente convergente en $H^{1}$ y tal que también $A^{n} \operatorname{grad} u_{n}$ converge débilmente en $L^{2}\left(\Omega, \mathbb{R}^{N}\right)$ y entonces, usando el Div-Curl lema, se demuestra a continuación que las siguientes sucesiones no oscilan con respecto a $x_{1}$ :

$$
\left(A^{n} \operatorname{grad} u_{n}\right)_{1}, \frac{\partial u_{n}}{\partial x_{2}}, \ldots, \frac{\partial u_{n}}{\partial x_{N}} .
$$

\section{- Afirmación 1:}

$\left(A^{n} \operatorname{grad} u_{n}\right)_{1}$, la primera componente de $A^{n} \operatorname{grad} u_{n}$, no oscila con respecto a $x_{1}$.

Demostración:

Sea $g_{n}\left(x_{1}\right) \rightarrow g_{\infty}\left(x_{1}\right)$ en $L^{\infty}$ débil $\star$. Llamemos

$$
D^{n}=A^{n} \operatorname{grad} u_{n} \quad y \quad E^{n}=g_{n}\left(x_{1}\right) e_{1},
$$

donde $e_{1}$ es el primer vector canónico en $\mathbb{R}^{N}$. Entonces se tendrá para todo $n$ que

$$
\operatorname{div} D^{n}=-f \quad y \quad \operatorname{curl} E^{n}=0
$$

y luego aplicando el Div-Curl lema se tiene

$$
\begin{aligned}
& \left(A^{n} \operatorname{grad} u_{n}\right)_{1}: g_{n}\left(x_{1}\right)=\left(D^{n}, E^{n}\right) \rightarrow\left(D^{\infty}, E^{\infty}\right) \\
= & \left(\mathrm{A}^{\text {ef }} \operatorname{grad} u_{\infty}\right)_{1}: g_{\infty}\left(x_{1}\right) .
\end{aligned}
$$

- Afirmación 2:

$$
\frac{\partial u_{n}}{\partial x_{i}} \text { no oscila con respecto a } x_{1} \text { si } i \geq 2 \text {. }
$$

Demostración:

Sea $g_{n}\left(x_{1}\right) \rightarrow g_{\infty}\left(x_{1}\right)$ en $L^{\infty}$ débil $\star$. Llamemos

$$
D^{n}=g_{n}\left(x_{1}\right) e_{i} \quad y \quad E^{n}=\operatorname{grad} u_{n},
$$


donde $e_{i}$ es el $i$-ésimo vector canónico en $\mathbb{R}^{N}$. Entonces se tendrá para todo $n$ que

$$
\operatorname{div} D^{n}=0 \quad y \quad \operatorname{curl} E^{n}=0
$$

y luego aplicando el Div-Curl lema se tiene que

$$
\frac{\partial u_{n}}{\partial x_{i}} g_{n}\left(x_{1}\right)=\left(D^{n}, E^{n}\right) \rightarrow\left(D^{\infty}, E^{\infty}\right)=\frac{\partial u_{\infty}}{\partial x_{i}} g_{\infty}\left(x_{1}\right)
$$

\section{- Fórmulas para materiales laminados:}

Supongamos ahora que estamos hablando de un material laminado, es decir tal que

$$
A^{n}\left(x_{1}\right)=\chi_{n}\left(x_{1}\right) A+\left(1-\chi_{n}\left(x_{1}\right)\right) B
$$

donde $\chi_{n}$ es la función característica del subconjunto de $\Omega$ donde en la etapa $n$ se usa el material con tensor $A$ y $\chi_{n}\left(x_{1}\right) \rightarrow \theta$ en $L^{\infty}$ débil $\star$ y, si existen $\alpha, \beta \in \mathbb{R}$ tales que para todo $n \in \mathbb{N} A^{n} \in \mathcal{M}(\alpha, \beta, \Omega)$, existirá una subsucesión convergente en el sentido H. Entonces si definimos un nuevo vector:

$$
N^{n}=\left(\begin{array}{c}
\left(A^{n} \operatorname{grad} u_{n}\right)_{1} \\
\frac{\partial u_{n}}{\partial x_{2}} \\
\cdot \\
\cdot \\
\cdot \\
\frac{\partial u_{n}}{\partial x_{N}}
\end{array}\right)
$$

de las componentes no oscilantes y otro vector con las componentes que si podrían oscilar con respecto a $x_{1}$

$$
O^{n}=\left(\begin{array}{c}
\frac{\partial u_{n}}{\partial x_{1}} \\
\left(A^{n} \operatorname{grad} u_{n}\right)_{2} \\
\cdot \\
\cdot \\
\cdot \\
\left(A^{n} \operatorname{grad} u_{n}\right)_{N}
\end{array}\right)
$$

uno puede reescribir la relación $F^{n}=A^{n}\left(x_{1}\right) \operatorname{grad} u_{n}$ como:

$$
O^{n}=B^{n}\left(x_{1}\right) N^{n}
$$


pero aquí uno puede tomar límite en la topología débil de $L^{2}$ y obtener una relación

$$
O^{\infty}=B^{\infty}\left(x_{1}\right) N^{\infty}
$$

de donde uno despeja

$$
F^{\infty}=A^{e f} \operatorname{grad} u_{\infty}
$$

y obtiene así fórmulas explícitas para cada una de las componentes de $A^{e f}$.

\section{3.- El caso de difusión}

A comienzos de los años sesenta los físicos Hashin y Shtrikman [9] derivaron cotas para la conductividad térmica de un material isotrópico construído a partir de mezclar dos materiales isotrópicos. A pesar de que la derivación de estas cotas no es matemáticamente rigurosa, ellas resultan ser óptimas, lo cual se demuestra aplicando el método antes expuesto.

Si aplicamos el esquema anterior al caso de la ecuación de difusión, en que queremos laminar dos materiales isotrópicos, es decir

$$
A=a I \quad y \quad B=b I,
$$

y digamos que $a<b$, pero sólo disponemos de una cantidad limitada del mejor conductor, de forma que tan solo podemos llenar con éste una fracción $1-\theta$ del volumen de $\Omega$, una laminación será un límite de tensores de la forma

$$
A^{n}\left(x_{1}\right)=\left(a \chi_{n}\left(x_{1}\right)+b\left(1-\chi_{n}\left(x_{1}\right)\right)\right) I
$$

donde $\chi_{n} \rightarrow \theta$ débil $\star$ en $L^{\infty}(\Omega)$.

Aplicando entonces el procedimiento de las fórmulas para materiales laminados, se tiene que

$$
F_{1}^{n}=\left(a \chi_{n}\left(x_{1}\right)+b\left(1-\chi_{n}\left(x_{1}\right)\right)\right) \frac{\partial u_{n}}{\partial x_{1}}
$$




$$
F_{i}^{n}=\left(a \chi_{n}\left(x_{1}\right)+b\left(1-\chi_{n}\left(x_{1}\right)\right)\right) \frac{\partial u_{n}}{\partial x_{i}} \quad \text { para } i=2, . ., N .
$$

Pero como las cantidades no oscilantes con respecto a $x_{1}$ son: $F_{1}^{n}$, $\frac{\partial u_{n}}{\partial x_{2}}, \ldots, \frac{\partial u_{n}}{\partial x_{N}}$, las ecuaciones anteriores deben reescribirse como

$$
\begin{gathered}
\frac{\partial u_{n}}{\partial x_{1}}=\frac{1}{a \chi_{n}\left(x_{1}\right)+b\left(1-\chi_{n}\left(x_{1}\right)\right)} F_{1}^{n}=\left(\frac{\chi_{n}\left(x_{1}\right)}{a}+\frac{1-\chi_{n}\left(x_{1}\right)}{b}\right) F_{1}^{n} \\
F_{i}^{n}=\left(a \chi_{n}\left(x_{1}\right)+b\left(1-\chi_{n}\left(x_{1}\right)\right)\right) \frac{\partial u_{n}}{\partial x_{i}} \quad \quad \text { para } i=2, . ., N .
\end{gathered}
$$

donde sí podemos pasar al límite en los productos, debido a que en ellos aparecen cantidades que no oscilan con repecto a $x_{1}$ multiplicadas por funciones que sólo dependen de $x_{1}$ y que convergen en $L^{\infty}$ débil $\star$. De esta forma obtenemos, en el límite cuando $n \rightarrow \infty$, las ecuaciones siguientes

$$
\begin{gathered}
\frac{\partial u_{\infty}}{\partial x_{1}}=\left(\frac{\theta}{a}+\frac{1-\theta}{b}\right) F_{1}^{\infty} \\
F_{i}^{\infty}=(a \theta+b(1-\theta)) \frac{\partial u_{\infty}}{\partial x_{i}} \quad \text { para } i=2, . ., N .
\end{gathered}
$$

Finalmente a partir de estas ecuaciones volvemos a escribir las relaciones usuales, desde donde podremos leer las componentes del tensor efectivo de difusión, pues obtenemos

$$
\begin{gathered}
F_{1}^{\infty}=\frac{a b}{\theta b+(1-\theta) a}: \frac{\partial u_{\infty}}{\partial x_{1}} \\
F_{i}^{\infty}=(a \theta+b(1-\theta)) \frac{\partial u_{\infty}}{\partial x_{i}} \quad \text { para } i=2, . ., N .
\end{gathered}
$$

Por tanto

$$
A^{e f}=\left[\begin{array}{cccc}
a_{-}(\theta) & 0 & \ldots \ldots & 0 \\
0 & a_{+}(\theta) & \ldots \ldots & 0 \\
\cdot & \cdot & \ldots \ldots \ldots \ldots \ldots & \cdot \\
0 & 0 & 0 & a_{+}(\theta)
\end{array}\right]
$$

donde

$$
\frac{1}{a_{-}(\theta)}=\frac{\theta}{a}+\frac{1-\theta}{b} \quad y \quad a_{+}(\theta)=\theta a+(1-\theta) b .
$$


Si uno itera el proceso de laminación, es decir, continúa relaminando los materiales obtenidos entre ellos, manteniendo fija la proporción $\theta$ que se usa del material $a$, se puede demostrar, ver Murat y Tartar [12], que uno genera el siguiente conjunto de tensores de difusión

$\mathcal{D}(a, b, \theta)=\left\{\begin{array}{ll}\sum_{i=1}^{N} \frac{1}{\lambda_{i}-a} \leq \frac{1}{a_{-}(\theta)-a}+\frac{N-1}{a_{+}(\theta)-a} \\ & \sum_{i=1}^{N} \frac{1}{b-\lambda_{i}} \leq \frac{1}{b-a_{-}(\theta)}+\frac{N-1}{b-a_{+}(\theta)}\end{array}\right\}$

Las cotas de Hashin-Shtrikman se deducen al considerar los materiales isotrópicos que están justo en el borde del conjunto $\mathcal{D}(a, b, \theta)$.

Los problemas de caracterizar el conjunto de tensores que se pueden generar a partir de laminar un material isotrópico con uno anisotrópico, o incluso a partir de dos materiales anisotrópicos, están todavía abiertos.

\section{4.- El caso de Elasticidad Lineal}

Trabajaremos con el sistema de elasticidad lineal y nuevamente con condición de borde de Dirichlet, es decir

$$
\left.\begin{array}{r}
-\operatorname{div}:(\mathcal{C}(x): \varepsilon(x):)=f(x) \quad x \in \Omega \\
u(x)=0 \quad x \in \partial \Omega,
\end{array}\right\}
$$

donde $u \in H_{0}^{1}\left(\Omega, \mathbb{R}^{N}\right)$ es el campo vectorial de desplazamientos y $\varepsilon \in L^{2}\left(\Omega, S y m_{N}\right)$ será el campo tensorial del gradiente de deformación simetrizado que se calcula como

$$
\varepsilon_{i j}=\frac{1}{2}\left(\frac{\partial u_{i}}{\partial x_{j}}+\frac{\partial u_{j}}{\partial x_{i}}\right) .
$$

$f \in H^{-1}\left(\Omega, \mathbb{R}^{N}\right)$ es la suma de las fuerzas externas aplicadas sobre el cuerpo $\Omega$. $\mathcal{C} \in L^{\infty}\left(\Omega, \mathbb{R}^{N^{4}}\right)$ es el tensor de elasticidad, cuyas componentes $C_{i j k l}$ satisfacen las siguientes simetrías:

$$
C_{i j k l}=C_{j i k l}=C_{i j l k}=C_{k l i j}
$$


La componente $i, j$ del esfuerzo interno o stress se calcula como

$$
\sigma_{i j}=\sum_{k, l=1}^{N} C_{i j k l} \varepsilon_{k l}
$$

y estas ecuaciones se denominan relaciones de deformación-esfuerzo.

Muchas veces necesitamos considerar el caso particular de materiales isotrópicos, y en este caso las componentes del tensor de elasticidad vienen dadas por

$$
C_{i j k l}(x)=\lambda(x) \delta_{i j} \delta_{k l}+\mu(x)\left(\delta_{i k} \delta_{j l}+\delta_{i l} \delta_{j k}\right),
$$

donde $\lambda(x)$ y $\mu(x)$ se denominan los parámetros de Lamé del material en la posición $x, x \in \Omega$, y $\delta_{i j}$ es el delta de Kronecker. En este caso las relaciones de deformación-esfuerzo pueden escribirse como

$$
\sigma_{i j}=\lambda \delta_{i j}: \operatorname{tr} \varepsilon+2 \mu \varepsilon_{i j} .
$$

Existen dos clases de tensores de elasticidad. Un tensor $\mathcal{C}$ se dice fuertemente elíptico si existe un escalar $\alpha>0$ tal que

$$
(\mathcal{C} \eta \otimes \xi, \eta \otimes \xi) \geq \alpha\|\eta\|^{2}\|\xi\|^{2} \quad \text { para todo } \eta, \xi \in \mathbb{R}^{N},
$$

donde (, ) es el producto interno usual de matrices y $\eta \otimes \xi=\eta \xi^{T}$. En el caso isotrópico estas condiciones son: $\mu>0$ y $2 \mu+\lambda>0$.

Un tensor de elasticidad $\mathcal{C}$ se dice muy fuertemente elíptico si existe un escalar $\alpha>0$ tal que

$$
(\mathcal{C} M, M) \geq \alpha\|M\|^{2} \quad \text { para todo } M \in \text { Sym }_{N} .
$$

En el caso isotrópico ésto se reduce a: $\mu>0$ y $2 \mu+N \lambda>0$.

Como era de esperar, elipticidad muy fuerte implica elipticidad fuerte pues, gracias a las simetrías asumidas en el tensor de elasticidad, puede reemplazarse en la definición de elipticidad fuerte $\eta \otimes \xi$ por su simetrización $\eta \otimes_{s} \xi=\frac{1}{2}(\eta \otimes \xi+\xi \otimes \eta)$. 
La condición de elipticidad fuerte es necesaria para que un tensor de elasticidad pueda ser aceptado físicamente, puesto que se deduce a partir de imponer que todas las ondas elásticas puedan propagarse con velocidad finita en cualquier dirección dentro del material y que puedan oscilar en cualquier sentido, ver Gurtin [7].

Bajo el supuesto de elipticidad muy fuerte se tiene que el sistema de elasticidad lineal tiene solución única y por tanto para un lado derecho fijo $f$ se tiene una sucesión acotada de soluciones para la sucesión de problemas que se deben resolver para calcular el límite $\mathcal{C}^{e f}$ en sentido $\mathrm{H}, \mathrm{y}$ de esta forma se tienen subsucesiones $\left\{u_{n}\right\} \subset H_{0}^{1}\left(\Omega, \mathbb{R}^{N}\right)$ y $\left\{\mathcal{C}^{n} \varepsilon^{n}\right\} \subset L^{2}\left(\Omega, S y m_{N}\right)$ que convergen débilmente y por tanto, de manera muy similar al método empleado en el caso de la ecuación de difusión, se puede probar un teorema de compacidad para la convergencia $\mathrm{H}$, y luego $\mathcal{C}^{e f}$ va a ser necesariamente muy fuertemente elíptico, ver Francfort-Murat [5]. En el caso más general de elipticidad fuerte este acotamiento no está garantizado y, más aún, en Le Dret [10] se construye un ejemplo de un cuerpo fuerte, pero no muy fuertemente elíptico, tal que para una fuerza externa dada, tiene un conjunto no acotado de soluciones del problema de Dirichlet. Originalmente se requería que $2 \mu+\lambda \approx 0$, sin embargo uno puede obtener una condición menos exigente para la misma construcción, en Gutiérrez [8] se obtuvo la condición

$$
\mu+\lambda<0
$$

la que, como se verá más adelante, reaparece cuando calculamos explícitamente el tensor efectivo obtenido al laminar.

Dado que en el caso muy fuertemente elíptico todo funciona bien porque

$$
(\mathcal{C} M, M) \geq \alpha\|M\|^{2} \quad \text { para todo } M \in \text { Sym }_{N},
$$

lo cual implica que el funcional de energía elástica

$$
\mathcal{E}(u)=\int_{\Omega}(\mathcal{C} \varepsilon, \varepsilon) d x
$$


es convexo, surge la idea de buscar formas de convexificar la energía elástica de un material que tan solo satisfaga la condición de elipticidad fuerte. Una forma de hacerlo es utilizando el concepto de lagrangeanos nulos, lo que corresponde a los subdeterminantes de la matriz grad u, ver Ball [1] por ejemplo, lo que en el caso planar (es decir $N=2$ ) nos lleva a considerar el siguiente funcional

$$
\mathcal{T}(u)=\mathcal{E}(u)+c \int_{\Omega}\left(\frac{\partial u_{1}}{\partial x_{1}} \frac{\partial u_{2}}{\partial x_{2}}-\frac{\partial u_{1}}{\partial x_{2}} \frac{\partial u_{2}}{\partial x_{1}}\right) d x
$$

donde $c$ es una constante que se elige apropiadamente, pues puede probarse que si $\mathcal{T}$ es convexo, el material homogenizado será necesariamente fuertemente elíptico. En el caso de una mezcla cualquiera de dos materiales isotrópicos: material $a$ que es fuertemente elíptico, pero no muy fuertemente elíptico, es decir con

$$
\mu_{a}>02 \mu_{a}+\lambda_{a}>0, \quad \mu_{a}+\lambda_{a} \leq 0
$$

y material $b$ que es muy fuertemente elíptico, es decir

$$
\mu_{b}>0 \mu_{b}+\lambda_{b}>0
$$

se tiene que el funcional $\mathcal{T}$ será convexo si y sólo si la siguiente desigualdad se satisface

$$
\mu_{b}>-\mu_{a}-\lambda_{a}
$$

Para el caso tridimensional, el resultado similar es un poco más complicado pues necesitamos considerar los siguientes lagrangeanos nulos

$\mathrm{y}$

$$
\begin{aligned}
J_{1}(u) & =\frac{\partial u_{2}}{\partial x_{2}} \frac{\partial u_{3}}{\partial x_{3}}-\frac{\partial u_{2}}{\partial x_{3}} \frac{\partial u_{3}}{\partial x_{2}} \\
J_{2}(u) & =\frac{\partial u_{1}}{\partial x_{1}} \frac{\partial u_{3}}{\partial x_{3}}-\frac{\partial u_{1}}{\partial x_{3}} \frac{\partial u_{3}}{\partial x_{1}}
\end{aligned}
$$

$$
J_{3}(u)=\frac{\partial u_{1}}{\partial x_{1}} \frac{\partial u_{2}}{\partial x_{2}}-\frac{\partial u_{1}}{\partial x_{2}} \frac{\partial u_{2}}{\partial x_{1}} .
$$

La condición obtenida en este caso es

$$
\mu_{b}>-\frac{1}{4}\left(2 \mu_{a}+3 \lambda_{a}\right)
$$


y, nuevamente, en este caso el material homogenizado será necesariamente fuertemente elíptico.

En parte debido a esta imposibilidad de poder convexificar la energía elástica para cualquier par de materiales, surge de manera natural la idea de calcular explícitamente el tensor homogenizado. Se debe dejar en claro, sin embargo, que dado que no se tienen las condiciones apropiadas en el tensor de elasticidad que se homogeniza, uno no necesa-riamente dispone a priori de subsucesiones débilmente convergentes, pues, como muestra el ejemplo de Le Dret, la solución no necesariamente está acotada en $H^{1}$ y por tanto al hacer el cálculo fuera del contexto de elipticidad muy fuerte, necesitamos suponer la e-xistencia de una sucesión de deformaciones $\left\{u_{n}\right\}$ que converge debilmente en $H^{1}\left(\Omega, \mathbb{R}^{N}\right)$ y tal que también la sucesión correspondiente de stresses $\left\{\mathcal{C}^{n} \varepsilon_{n}\right\}$ converge débilmente en $L^{2}\left(\Omega\right.$, Sym $\left._{N}\right)$, este supuesto se justifica en la aproximación de deformaciones pequeñas en que se basa la derivación del sistema de elasticidad lineal, ver Gurtin [7]. De esta forma se obtuvieron los siguientes resultados:

i) Si los parámetros de Lamé del material no muy fuertemente elíptico satisfacen:

$$
2 \mu+N \lambda<0,
$$

se pueden seleccionar materiales muy fuertemente elípticos, de forma tal que al tomar el límite en sentido H de la sucesión de tensores de elasticidad correspondientes a sucesivas laminaciones, el tensor resultante no será fuertemente elíptico, lo cual contradice el requerimiento básico que todo tensor de elasticidad debe satisfacer para ser considerado realista. Si $\mu+\lambda<0$ basta con una laminación, en cambio si $\mu+\lambda \geq 0$ se necesitan dos laminaciones para conseguir este resultado.

ii) Si los parámetros de Lamé del material no muy fuertemente elíptico satisfacen:

$$
2 \mu+N \lambda=0,
$$

entonces para cualquier material isotrópico muy fuertemente elíptico con el cual se haga una laminación, el material resultante será también muy fuertemente elíptico. 
Los detalles de la obtención de estos resultados se presentan en Gutiérrez [8] y a la luz de la afirmación $i$ ) uno debe preguntarse si la explicación de este comportamiento contradictorio con la realidad física es simplemente que no existen materiales tales que $2 \mu+N \lambda<0$ ó bien que la teoría de elasticidad lineal no es lo suficientemente buena como para poder hacer homogenización en el contexto más general que ella, en principio, permite.

Quedan, por cierto, una infinidad de preguntas interesantes por estudiar, como por ejemplo:

i.- Dado que la noción de convergencia H es local, uno podría esperar que existan formas de convexificar la energía elástica en el caso de trabajar con condiciones de borde de tipo Neumann o de tipo mixto.

ii.- Un estudio análogo pero para el caso de tensores de elasticidad anisotrópicos, para el cual el cálculo explícito se torna extremadamente engorroso.

iii.- Un estudio del comportamiento de las ondas elásticas al tomar límite en sentido $\mathrm{H}$ para los casos en que se genera un tensor no fuertemente elíptico.

Es interesante comparar los resultados aquí esbozados, con sus similares pero obtenidos usando otras técnicas. Empleando $\Gamma$ convergencia, la cual se basa más bien en estudiar el problema de minimización de energía, en lugar de estudiar las ecuaciones dife-renciales parciales directamente, se estudia en Geymonat-Müller-Triantafyllidis [6], entre otras cosas, el caso de la frontera que separa el conjunto de los tensores muy fuertemente elípticos del resto de los tensores fuertemente elípticos en el caso anisotrópico. Cuando se reducen sus resultados al caso isotrópico, ellos están en completo acuerdo con los aquí obtenidos, siendo los nuestros más precisos en algunas situaciones, debido a que las fórmulas que ellos obtienen no son explícitas. Por otro lado en Francfort-Milton [4] se estudia el problema de caracterizar los conjuntos de tensores que son estables bajo laminación, es decir que al 
laminar dos materiales cuyos tensores estén en ese conjunto, el tensor homogenizado siga estando allí. La caracterización que ellos obtienen es sin embargo no explícita, lo cual dificulta su aplicación. Nuestros cálculos muestran que en el caso de elasticidad lineal, un tal conjunto no puede contener la clase de los tensores muy fuertemente elípticos y también un tensor isotrópico tal que $2 \mu+N \lambda<0$.

\section{Bibliografía}

1. J. M. Ball: Constitutive Inequalities and Existence Theorems in Nonlinear Elastostatics. Nonlinear Analysis and Mechanics: Heriot-Watt Symposium, Vol. I. ed. R. J. Knops, Pitman Res. Notes Math. 17,187-241 (1977).

2. J. M. Ball: A Version of the Fundamental Theorem for Young Measures. PDEs and Continuum Models of Phase Transitions. Lecture Notes in Physics vol. 344, eds. M. Rascle et al. SpringerVerlag (1989).

3. G. Dal Maso: An Introduction to $\Gamma$-Convergence. Progress in Nonlinear Differential Equations and their Applications, vol. 8, Birkhäuser, Boston (1993).

4. G. Francfort y G. Milton: Sets of Conductivity and Elasticity Tensors Stable under Lamination. Comm. Pure Appl. Math. XLVII, 257-279 (1994).

5. G. Francfort y F. Murat: Homogenization and Optimal Bounds in Linear Elasticity. Arch. Rat. Mech. Anal. 94, 307-334 (1986).

6. G. Geymonat, S. Müller y N. Triantafyllidis: Homogenization of Nonlinearly Elastic Materials, Mic roscopic Bifurcation and Macroscopic Loss of Rank-One Convexity. Arch. Rational Mech. Anal. 122, 231-290 (1993).

7. M. Gurtin: The Linear Theory of Elasticity. Handbuch der Physik Vol. VIa/2. Springer-Verlag (1972). 
8. S. Gutiérrez: Laminations in Linearized Elasticity: The Isotropic Non-very Strongly Elliptic Case. J. Elasticity 53, 215-256 (1998).

9. Z. Hashin y S. Shtrikman: A variational approach to the theory of effective magnetic permeability of multiphase materials. J. Applied Physics 33, 3125-3131 (1962).

10. H. Le Dret: An Example of $H^{1}$-unboundedness of Solutions to Strongly Elliptic Systems of Partial Differential Equations in a Laminated Geometry. Proc. Roy. Soc. Edinburgh 105A, 77-82 (1987).

11. F. Murat: H-convergence. Séminaire d'Analyse Fonctionnelle et Numérique de l'Université d'Alger (1977).

12. F. Murat y L. Tartar: Calculus of Variations and Homogenization. Collection d'Etudes de Electricité de France (1983).

13. P. Pedregal: Parametrized Measures and Variational Principles. Progress in Nonlinear Differential Equations and their Applications, vol. 30, Birkhäuser, Basel (1997).

14. S. Spagnolo: Sulla convergenza di soluzioni di equazioni paraboliche ed ellitiche. Ann. Sc. Norm. Sup. di Pisa Cl. di Scienze 22, 577-597 (1968).

15. L. Tartar: Cours Peccot, Collège de France (1977).

16. L. Tartar: Compensated Compactness and applications to Partial Differential Equations. Nonlinear Analysis and Mechanics: Heriot Watt Symposium, vol. IV, ed. R.J. Knops. Research Notes in Mathematics 39, Pitman 136-212 (1979).

17. L. Tartar: Homogenization, Compensated Compactness and HMeasures. CBMS-NSF Conference, Santa Cruz, June 1993. Notas en preparación.

Received : October 2000. 


\section{Sergio Gutiérrez}

Centro Anestoc

Facultad de Matemáticas

Pontificia Universidad Católica de Chile

Casilla 306

Santiago 22

Chile

e-mail : sgutierr@mat.puc.cl. 